\title{
Corrigendum: The energetic and carbon economic origins of leaf thermoregulation
}

Sean T. Michaletz, Michael D. Weiser, Nate G. McDowell, Jizhong Zhou, Michael Kaspari, Brent R. Helliker and Brian J. Enquist

Nature Plants 2, 16129 (2016); published 22 August 2016; corrected 26 August 2016.

In the version of this Article originally published, the affiliation for Nate G. McDowell was incorrect. This has now been corrected. 